\title{
Salvianolic acid $B$ attenuates renal interstitial fibrosis by regulating the HPSE/SDC1 axis
}

\author{
YANG HU*, MAN WANG* , YUNZHENG PAN, QINGJU LI And LI XU \\ Jiangsu Key Laboratory for Pharmacology and Safety Evaluation of Chinese Materia Medica, \\ School of Pharmacy, Nanjing University of Chinese Medicine, Nanjing, Jiangsu 210023, P.R. China
}

Received December 16, 2019; Accepted May 28, 2020

DOI: $10.3892 / \mathrm{mmr} .2020 .11229$

\begin{abstract}
Salvianolic acid B (Sal B) is one of the main water-soluble components of Salvia miltiorrhiza Bge. Numerous reports have demonstrated that it could exert significant renal-protective effects, but the underlying mechanism remains unclear. The present study demonstrated that Sal B could alleviate renal injury by regulating the heparanase/syndecan-1 (HPSE/SDC1) axis. In vivo, the serum creatinine, blood urea nitrogen, transforming growth factor- $\beta 1$ (TGF- $\beta 1$ ) and fibroblast growth factor-2 (FGF-2) levels, and the histopathological changes of mice kidneys were examined. Sal B could notably reduce the renal injury caused by left ureteral ligation. In vitro, Sal B downregulated the expression levels of HPSE/FGF-2/TGF- $\beta 1 / \alpha$-smooth muscle actin and upregulated the expression levels of SDC1/E-cadherin in angiotensin II-stimulated HK-2 cells in a dose-dependent manner. In summary, to the best of the authors' knowledge, the present study provided evidence for the first time that Sal B could exert renal-protective effects via the inhibition of the HPSE/SDC1 axis, and these results suggest that the administration of Sal B may be a novel therapeutic strategy in treating renal interstitial fibrosis.
\end{abstract}

\section{Introduction}

Chronic kidney disease (CKD) is a long-term condition characterized by renal dysfunction that lasts 3 months (1). CKD remains a leading public health issue, and patients with CKD have poorer quality of life compared with those of healthy individuals (2). There are numerous causes of CKD, the most

Correspondence to: Professor Li Xu, Jiangsu Key Laboratory for Pharmacology and Safety Evaluation of Chinese Materia Medica, School of Pharmacy, Nanjing University of Chinese Medicine, 138 Xianlin Avenue, Qixia, Nanjing, Jiangsu 210023, P.R. China E-mail: xuli64@163.com

*Contributed equally

Key words: salvianolic acid B, renal interstitial fibrosis, heparanase, syndecan-1, angiotensin II typical of which are hypertension and type 2 diabetes (3). Although several studies on CKD have been performed $(4,5)$, the molecular mechanisms involved in the occurrence and development of this disease are not fully understood yet. Therefore, there is an urgent need to understand the mechanism of CKD and to identify appropriate strategies to manage it.

Renal interstitial fibrosis occurs in virtually every type of CKD, and the severity of tubulointerstitial fibrosis has long been considered a crucial factor of progressive renal injury in glomerulonephritis (6). The primary histopathological features of renal interstitial fibrosis include marked accumulation of fibroblasts and deposition of extracellular matrix (7). Several cellular pathways, including the mesangial and tubular epithelial-myofibroblast transdifferentiation (TEMT), have been identified as important in renal interstitial fibrosis (8). The main feature of TEMT is the fact that renal tubular epithelial cells acquire mesenchymal phenotypes and myofibroblast functions. The transdifferentiation of TEMT induces kidney epithelial cells to decrease the expression of adherens junction proteins, such as E-cadherin, and to promote the expression of numerous fibroblast markers, including $\alpha$-smooth muscle actin $(\alpha-S M A)$, transforming growth factor- $\beta 1$ (TGF- $\beta 1)$ and fibroblast growth factor-2 (FGF-2) (9). The inhibition of TEMT has also been considered as an effective way to attenuate renal interstitial fibrosis (10).

Heparan sulfate (HS) proteoglycans are important constituents of the cell membrane, and they act as co-receptors for cellular signaling. Cell surface HS proteoglycans are important regulators of cellular migratory, mitogenic, secretory and inflammatory activities (11). Syndecan-1 (SDC1) is a member of the syndecan family, which comprises a transmembrane HS proteoglycan. It can exert various biological functions at the surface of renal epithelial cells, and plays important roles in cell-cell or cell-matrix interactions (12). Heparanase (HPSE), as an endo- $\beta$-D-glucuronidase, can cleave HS at specific intrachain sites (13). Precious studies reported that increased levels of HPSE activity are associated with several diseases, such as renal fibrosis, cancer (14) and Alzheimer's disease (15). Several stimulating factors, such as ROS and angiotensin II (AngII), can promote the expression of HPSE, which in turn cleaves the HS side-chains of SDC1 and promotes TEMT, eventually leading to renal fibrosis (16).

Salvianolic acid B (Sal B) is a phenolic acid isolated from Salvia miltiorrhiza Bge. It exhibits various pharmacological 
activities, including antioxidant (17), anti-myocardial ischemia (18), antitumor (19), and anti-inflammatory (20) activities. A previous study has demonstrated thatSalB has kidney-protective effects (21). However, whether Sal B could reduce renal interstitial fibrosis by regulating the activation of the HPSE/SDC1 axis remains unknown. It was hypothesized that the mechanism of Sal B against renal interstitial fibrosis may be associated with the inhibition of the HPSE/SDC1 axis. Therefore, the present study was designed to investigate the renal-protective effects of Sal B and to explore its underlying mechanism.

\section{Materials and methods}

Materials. Sal B ( $\geq 98 \%$ purity) was provided by Nanjing Hongqiao Institute of Pharmaceutical Technology. Dulbecco's modified Eagle's medium/Ham's F12 (DMEM/F12) was purchased from Wisent, Inc. Fetal bovine serum (FBS) was purchased from Biological Industries. Trypsin was purchased from Beyotime Institute of Biotechnology. Serum creatinine (CR; cat. no. ANG-E12726H), blood urea nitrogen (BUN; cat. no. ANG-E12727H), TGF- $\beta 1$ (cat. no. ANG-E10095H) and FGF-2 (cat. no. ANG-E12728H) ELISA kits were purchased from AngleGene BioTechnology Co. Ltd, Nanjing, China. AngII was purchased from Tocris Bioscience. Primary antibodies against HPSE (cat. no. ab59787), SDC1 (cat. no. ab128936), TGF- $\beta 1$ (cat. no. ab92486), FGF-2 (cat. no. ab8880) and $\alpha$-SMA (cat. no. ab5694) were obtained from Abcam. Antibodies against E-cadherin (cat. no. 3195S) and GAPDH (cat. no. 5174s) were obtained from Cell Signaling Technology, Inc. The water used in this study was purified by a Milli- ${ }^{\circledR}$ water purification system (EMD Millipore). All culture plates were obtained from Corning, Inc.

Animals. A total of 35 2-month-old male C57BL/6 (C57) mice $(20 \pm 2 \mathrm{~g})$ were purchased from Beijing Vital River Laboratory Animal Technology Co., Ltd. The mice were housed under pathogen-free conditions under a 12-h light/dark cycle at $22-24^{\circ} \mathrm{C}$ and had ad libitum access to water and a regular pellet diet (22). The present study was carried out in accordance with the principles of the Basel Declaration and the recommendations of the Guidelines of Jiangsu Regulation for the Administration of Laboratory Animals, and the protocols were approved by the Animal Ethics Committee of Nanjing University of Chinese Medicine (approval no. ACU-2320151203).

Experimental protocol and drug administration. The mice were randomly divided into 5 groups ( $\mathrm{n}=7$ mice/group, the group size was decided based on the initial pilot study), including the sham, unilateral ureteral obstruction (UUO), UUO + Sal B $(6.25 \mathrm{mg} / \mathrm{kg}), \mathrm{UUO}+\mathrm{Sal}$ B $(12.5 \mathrm{mg} / \mathrm{kg})$ and $\mathrm{UUO}+\mathrm{Sal} \mathrm{B}$ $(25 \mathrm{mg} / \mathrm{kg})$ groups. The mice in the UUO and Sal B treatment groups were subjected to partial UUO operation according to a previous study (23). Briefly, sodium pentobarbital (50 mg/kg) was used to anesthetize the mice, and then the left ureter was isolated and a $0.20-\mathrm{mm}$ steel wire segment was placed next to the left ureter at the ureteropelvic junction. The wire was ligated with the ureter using a single 6-0 silk suture, and then the wire was removed. The mice in sham group underwent the same operation without ligation. After the operation, the mice in the Sal B treatment groups received a daily intraperitoneal injection of Sal B, while the mice in the sham and UUO groups received equal amounts of normal saline. All mice were sacrificed 14 days later. The anesthetic drug sodium pentobarbital $(50 \mathrm{mg} / \mathrm{kg})$ was used to anesthetize the mice, then the blood was collected from the mice retro-orbital sinus for a series of biochemical assays. Then, $\mathrm{CO}_{2}(20 \% \mathrm{~V} / \mathrm{min} ; 1.2 \mathrm{l} / \mathrm{min})$ was used to euthanize mice at the endpoint, and the kidneys were harvested for morphological and biochemical studies. Skillful operations were applied to reduce animal suffering during the experiment.

Histopathological observation. The kidneys were immersed in $10 \%$ formaldehyde solution for $24 \mathrm{~h}$ at room temperature immediately after isolation and then embedded in paraffin. Next, the kidney tissues were cut into $4-\mu$ m-thick sections (24). The tissue sections were stained with hematoxylin and eosin (H\&E) for $5 \mathrm{~min}$ or Masson's stain for $10 \mathrm{~min}$ at room temperature to observed the histopathological changes under a light microscope at x200 magnification.

Immunohistochemical analyses. Mice kidney sections were treated for 10 min with 3\% hydrogen peroxide/methanol and 30 min with 5\% BSA (Solarbio Life Sciences; cat. no. A8010) at room temperature. Sections were incubated overnight at $4^{\circ} \mathrm{C}$ with primary antibodies against HPSE $(1: 100)$ and SDC1 (1:100), and then labeled with streptavidin-peroxidase (1:50; cat. no. A0208; Beyotime Institute of Biotechnology) for $30 \mathrm{~min}$ at $37^{\circ} \mathrm{C}$, followed by $5 \min 3,3^{\prime}$-diaminobenzidine tetrahydrochloride solution staining, $8 \mathrm{~min}$ hematoxylin counterstaining and mounting using neutral balsam. Immunoreactive proteins were viewed with a light microscope at x200 magnification.

Determination of $C R, B U N, T G F-\beta 1$ and FGF-2 levels in serum. Blood was collected from the mice retro-orbital sinus on day 14 after surgery. Then, the serum CR, BUN, TGF- $\beta 1$ and FGF-2 levels were assessed with a microplate system (BioTek Instruments, Inc.) according to the manufacturer's instructions (25).

Cell culture and treatment. HK-2 cells were obtained from American Type Culture Collection and routinely cultured in DMEM/F12 supplemented with $10 \%$ FBS and incubated at $37^{\circ} \mathrm{C}$ with $5 \% \mathrm{CO}_{2}$.

HK-2 cells were seeded on a 6-well plate a density of $5 \times 10^{4}$ cells. Subsequently, $1 \mu \mathrm{M}$ AngII was added to stimulate the cells for $48 \mathrm{~h}$ at $37^{\circ} \mathrm{C}$. Then, cells were treated with different concentrations of Sal B $(0.1,1$ and $10 \mu \mathrm{M})$ for $24 \mathrm{~h}$ at $37^{\circ} \mathrm{C}$. The cells or the cell supernatants (centrifugation at $300 \mathrm{xg}$ at room temperature for $5 \mathrm{~min}$ ) were then collected for various analyses.

MTT assay. To determine the appropriate AngII treatment concentration, an MTT assay was performed. Briefly, HK-2 cells were seeded on a 96-well plate at a density of $1 \times 10^{4}$ cells. After culture overnight, different concentrations of AngII $(0,0.0001,0.001,0.01,0.1,1,10$ and $100 \mu \mathrm{M})$ were added to each well and stimulated for $48 \mathrm{~h}$ at $37^{\circ} \mathrm{C}$. Then, MTT $(5 \mathrm{mg} / \mathrm{ml})$ prepared with phosphate buffer (PBS) was added to each well $(10 \mu \mathrm{l})$. After incubation for $4 \mathrm{~h}$ at $37^{\circ} \mathrm{C}$, the supernatant of each 

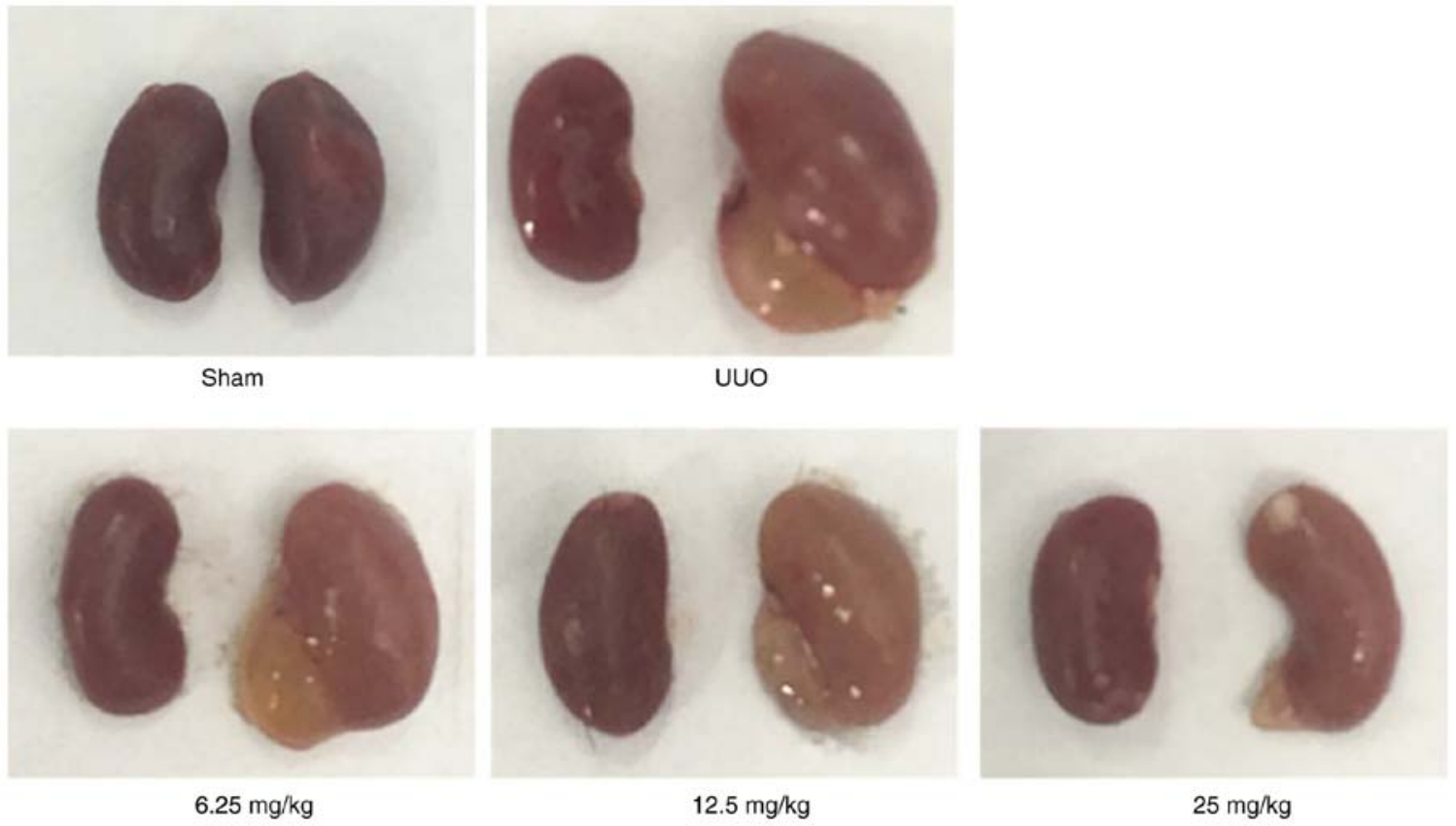

Figure 1. Effect of salvianolic acid B on the renal morphology of each group of mice ( $n=7$ mice/group). UUO, unilateral ureteral obstruction.

well was discarded and the formazan was dissolved in $150 \mu \mathrm{l}$ DMSO. Then, the absorbance at $570 \mathrm{~nm}$ was read by BioTek Synergy ${ }^{\mathrm{TM}} 2$ microplate reader (BioTek Instruments, Inc.) and the cell viability of each group was calculated. According to the experimental results, when the concentration of AngII was $\geq 10 \mu \mathrm{M}$, the viability of $\mathrm{HK}-2$ cells declined markedly, and AngII stimulation $(1 \mu \mathrm{M})$ could notably promote the expression of key regulators of TEMT, such as TGF- $\beta 1$ and FGF-2. So, $1 \mu \mathrm{M}$ was selected as the treatment concentration of AngII (data not shown).

Detection of TGF- $\beta 1$ and FGF-2 in cell supernatant. HK-2 cells were seeded on a 6 -well plate at a density of $5 \times 10^{4}$ cells. After overnight culture, AngII (final concentration, $1 \mu \mathrm{M}$ ) was added to each well and stimulated for $48 \mathrm{~h}$ at $37^{\circ} \mathrm{C}$. Subsequently, cells were treated with different concentrations of Sal B $(0.1,1$ and $10 \mu \mathrm{M})$ for $24 \mathrm{~h}$ at $37^{\circ} \mathrm{C}$, and the cell supernatant of each group was collected for analysis (centrifugation at $300 \mathrm{x} \mathrm{g}$ at room temperature for $5 \mathrm{~min}$ ).

Immunofluorescence. HK-2 cells seeded on glass coverslips in 24-well plates at a density of $2 \times 10^{4}$ cells were washed 3 times with PBS after treatment, and then fixed for $30 \mathrm{~min}$ with $4 \%$ paraformaldehyde at $37^{\circ} \mathrm{C}$. Next, cells were washed twice with PBS, incubated with $1 \%$ BSA (Beijing Solarbio Science \& Technology Co., Ltd., cat. no. A8010) for $1 \mathrm{~h}$ at room temperature, and incubated with a primary rabbit anti-HPSE (1:100) or anti-SDC1 (1:100) antibody in blocking buffer for $12 \mathrm{~h}$ at $4^{\circ} \mathrm{C}$. Then, cells were washed twice with PBS and incubated with DAPI-goat anti-rabbit antibody (1:500; Beijing Solarbio Science \& Technology Co., Ltd., cat. no. A0562) for $2 \mathrm{~h}$ at $37^{\circ} \mathrm{C}$. The images were acquired using a fluorescence microscope (26).

Western blotting. The kidney tissues (including both the medulla and cortex) and HK-2 cells were homogenized in ice-cold RIPA buffer containing $2 \mathrm{mM}$ PMSF. The samples were centrifuged at $12,000 \mathrm{x}$ for $15 \mathrm{~min}$ at $4^{\circ} \mathrm{C}$, and the concentrations of total protein were quantified using a NanoDrop instrument (NanoDrop Technologies; Thermo Fisher Scientific, Inc.). Total protein samples $(30 \mu \mathrm{g})$ were then separated by SDS-PAGE on 6-10\% gels and electro-transferred onto PVDF membranes. Then, the membranes were blocked with $5 \%$ BSA for $1 \mathrm{~h}$ at room temperature and incubated overnight at $4^{\circ} \mathrm{C}$ with primary antibodies against HPSE $(1: 1,000)$, SDC1 (1:1,000), FGF-2 (1:1,000), TGF- $\beta 1(1: 1,000)$, E-cadherin $(1: 1,000), \alpha$-SMA $(1: 1,000)$ and GAPDH $(1: 1,000)$. After washing 3 times with TBS-Tween-20 (1\%), membranes were incubated with horseradish peroxidase-conjugated secondary antibodies (1:1,000; Beijing Solarbio Science \& Technology Co., Ltd., cat. no. A0208) in 5\% BSA at room temperature for $1 \mathrm{~h}$ (27). The membranes were visualized using an ECL advanced kit and target proteins were detected with a gel imaging system (Image Lab software version 4.0.1; Bio-Rad Laboratories, Inc.).

Cell transfection. Plasmids for overexpression of HPSE and empty control plasmids were purchased from Shanghai GenePharma Co., Ltd. HK-2 cells were seeded on a 6-well plate at a density of $5 \times 10^{4}$ cells and divided into five groups, including the negative control (NC), Homo HPSE + AngII, Homo HPSE, NC + AngII and Homo HPSE + AngII + Sal B groups. Transient transfection was performed using Lipofectamine ${ }^{\circledR}$ 2000 (Thermo Fisher Scientific, Inc.) according to the manufacturer's protocol. A total of $3 \mu \mathrm{g}$ overexpressed RNA (final concentration, $3 \mu \mathrm{g} / \mathrm{ml}$ ), and $9 \mu 1$ Lipofectamine 2000 were added to the serum-free medium and incubated at $25^{\circ} \mathrm{C}$ for $20 \mathrm{~min}$. Then, the plasmids were mixed into per group and cultured in serum-free DMEM at $37^{\circ} \mathrm{C}$. After $6 \mathrm{~h}$ in culture, the medium was replaced by DMEM containing $10 \% \mathrm{FBS}$ and the cells were cultured for $24 \mathrm{~h}$ at $37^{\circ} \mathrm{C}$. 


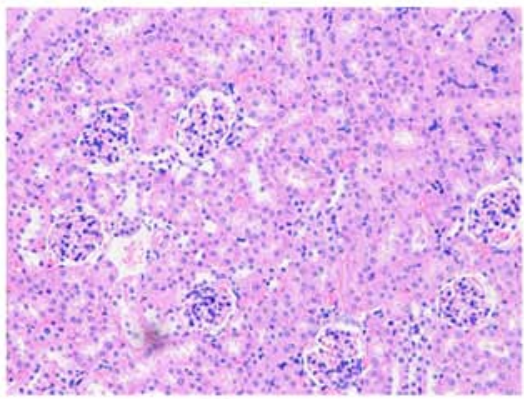

Sham

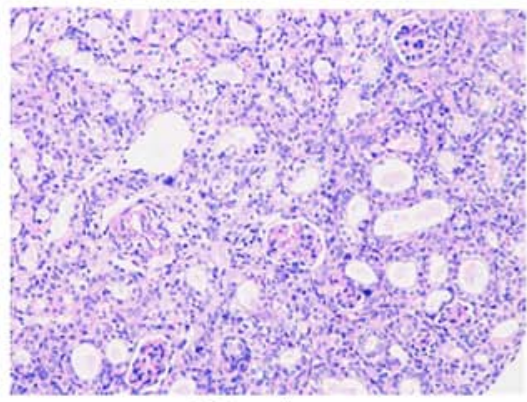

$6.25 \mathrm{mg} / \mathrm{kg}$

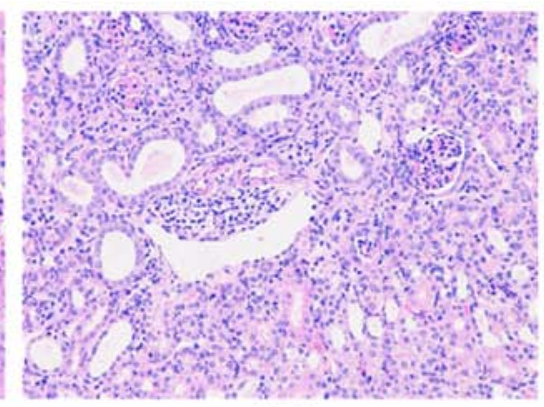

UUO

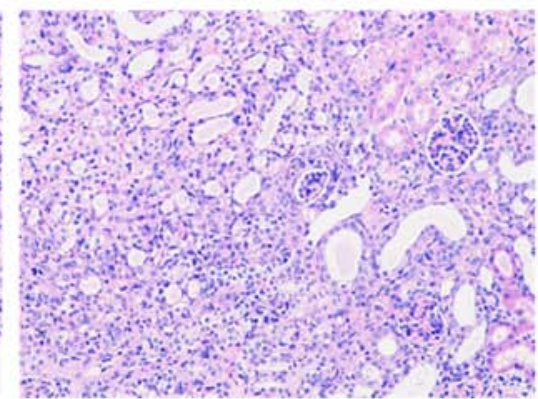

$12.5 \mathrm{mg} / \mathrm{kg}$

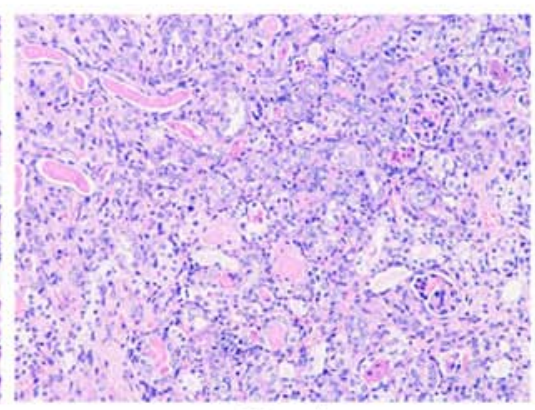

$25 \mathrm{mg} / \mathrm{kg}$

Figure 2. Histopathological observation of the kidneys in each group of mice ( $\mathrm{n}=3$ mice/group). In the sham group, the kidney tissues maintained a normal morphology and clear structure. In the UUO group, the kidney tissues showed marked cavitation in the renal tubular epithelium and evident inflammatory cell infiltration. In the Sal B $(6.25 \mathrm{mg} / \mathrm{kg})$ and Sal B $(12.5 \mathrm{mg} / \mathrm{kg})$ groups, the kidney tissues showed partial cavitation of the tubular epithelium with moderate inflammatory cell infiltration in the renal tubulointerstitium. In the Sal B $(25 \mathrm{mg} / \mathrm{kg})$ group, the tubular epithelial cavitation and inflammatory cell infiltration were notably alleviated (magnification, x200). Sal B, salvianolic acid B; UUO, unilateral ureteral obstruction.

Statistical analysis. Data are presented as the mean \pm SD. The statistical significance of differences between the means of multiple groups was analyzed by one-way ANOVA, followed by Tukey's multiple comparison test. $\mathrm{P}<0.05$ was considered to indicate a statistically significant difference.

\section{Results}

Effect of Sal B on renal morphology in mice with renal fibrosis. To explore the protective effects of Sal B on UUO-induced mice kidneys, 3 mice were randomly selected 14 days after modelling. As shown in Fig. 1, the sizes of the kidney in the UUO group were markedly increased after left ureteral ligation, whereas Sal B treatment notably reduced the kidney sizes.

Sal B alleviates the pathological changes of mice kidneys. The results of H\&E staining demonstrated that the kidneys of the mice in the sham group maintained a normal morphology and clear structure with no interstitial edema, while those of the mice in the UUO group showed notable cavitation in the renal tubular epithelium, evident inflammatory cell infiltration in the renal tubulointerstitium and distinct tubular expansion. These conditions could notably be restored by Sal B treatment (Fig. 2). Besides, the result of Masson's staining showed that there were numerous collagen fiber streaks stained blue with obvious collagen fiber hypertrophy in the UUO group. However, fewer collagen fiber streaks were observed in the Sal B treatment groups. These results indicated that Sal B could alleviate renal injury and fibrosis in fibrotic mice (Fig. 3).
Effects of Sal B on BUN/CR in the serum of renal fibrotic mice. The serum levels of BUN and CR are the classical markers of renal function (28). To evaluate the efficacy of Sal B on renal function after left ureteral ligation, the expression levels of BUN and CR in serum were determined in the present study. The results showed that left ureteral ligation resulted in a significant increase in the levels of BUN and CR (Fig. 4), but the administration of Sal B (12.5 and $25 \mathrm{mg} / \mathrm{kg}$ ) notably reduced the expression of these cytokines.

Sal B regulates the expression levels of HPSE and SDC1 in mice kidneys. Using immunohistochemical analysis, the effect of Sal B on the expression of HPSE and SDC1 in kidney tissues was examined. As shown in Fig. 5, left ureteral ligation notably downregulated the expression level of HPSE and upregulated SDC1. However, these changes were notably altered by Sal B treatment.

Sal B inhibits TEMT in renal fibrotic mice. To evaluate whether Sal B could affect the expression levels of fibrosis markers, ELISA and western blotting were performed. The primary feature of renal interstitial fibrosis is the accumulation of extracellular matrix components. Myofibroblasts are considered to be primary renal interstitial cells, which can produce large quantities of extracellular matrix during fibrosis (29). Furthermore, the activation of TEMT is closely associated with the production of myofibroblasts (30). The present results showed that Sal B could significantly downregulate the expression level of $\alpha$-SMA and upregulate the protein level of E-cadherin compared with the UUO group (Fig. 6). TGF- $\beta 1$ and FGF-2 are key regulators of the TEMT 


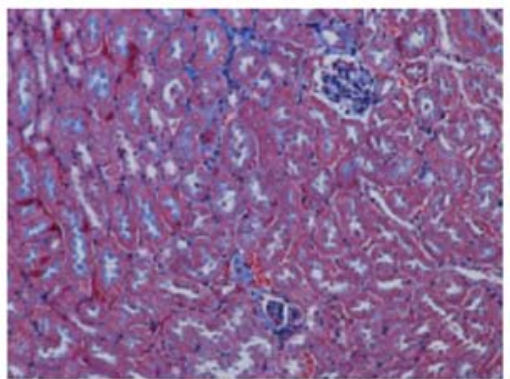

Sham

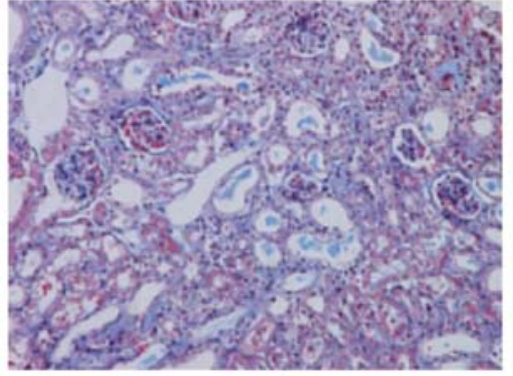

$6.25 \mathrm{mg} / \mathrm{kg}$

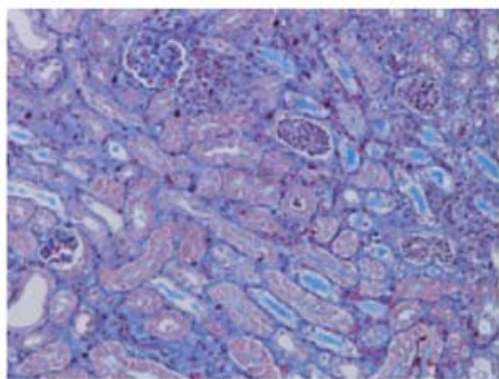

UUO

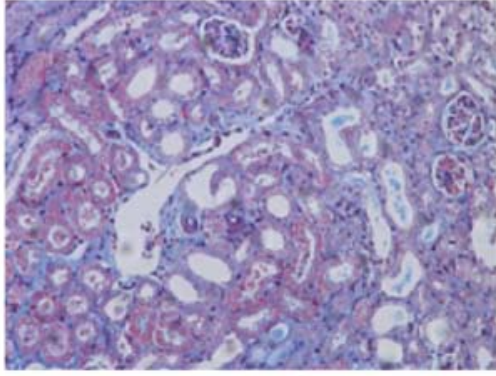

$12.5 \mathrm{mg} / \mathrm{kg}$

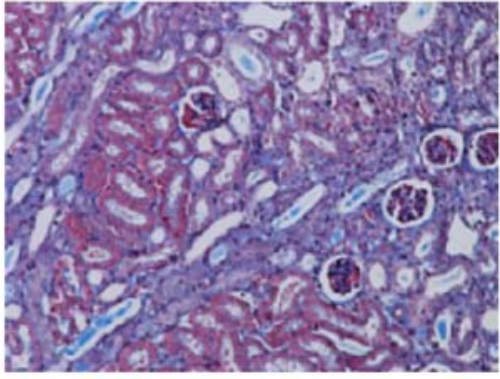

$25 \mathrm{mg} / \mathrm{kg}$

Figure 3. Representative images of Masson's staining of the kidneys of mice in each group ( $\mathrm{n}=3$ mice/group). The UUO group showed numerous collagen fiber streaks with obvious collagen fiber hypertrophy, but these conditions were notably alleviated by salvianolic acid B treatment (magnification, x200). UUO, unilateral ureteral obstruction.
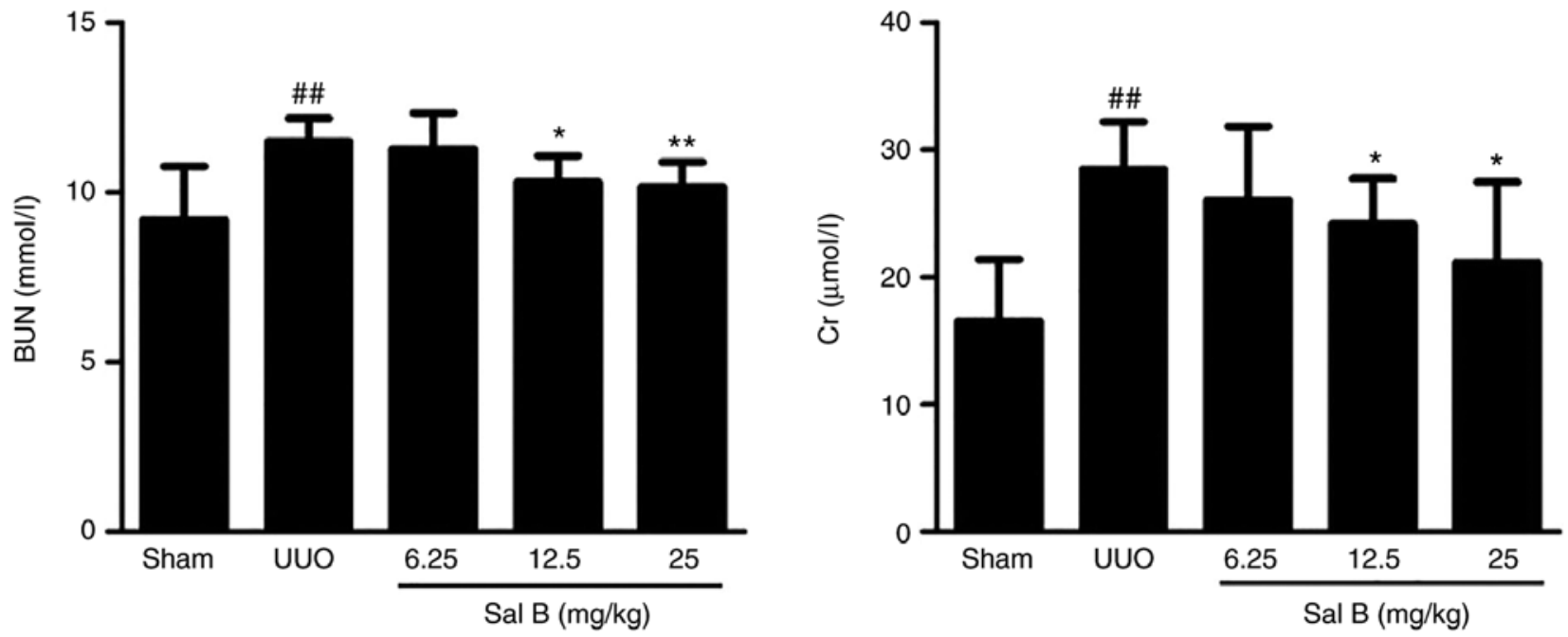

Figure 4. Effects of Sal B on BUN/CR in serum (n=3 mice/group). Mice were intraperitoneally injected with Sal B after left ureteral ligation. Data are expressed as the mean \pm SD. ${ }^{*} \mathrm{P}<0.05$ and ${ }^{* *} \mathrm{P}<0.01$ vs. UUO group; ${ }^{\# \#} \mathrm{P}<0.01$ vs. sham group. Sal $\mathrm{B}$, salvianolic acid $\mathrm{B}$; UUO, unilateral ureteral obstruction.

process (31), the present study demonstrated that Sal B attenuated the UUO-induced upregulation of these proteins in UUO mice (Figs. 6 and 7).

Effects of Sal B on TGF- $\beta 1 / F G F-2$ in the supernatant of $H K-2$ cells. TGF- $\beta 1$ and FGF-2 are key regulators of TEMT (32). To evaluate the efficacy of Sal B on TEMT, the expression levels of TGF- $\beta 1$ and FGF- 2 in HK- 2 cell supernatant were determined. The results showed that AngII stimulation significantly increased the expression levels of TGF- $\beta 1$ and FGF-2 (Fig. 8). However, Sal B treatment (1 and $10 \mu \mathrm{M})$ significantly reduced the expression levels of these cytokines.
Sal B suppresses renal interstitial fibrosis by inhibiting the HPSE/SDC1 axis. To explore the underlying mechanisms of Sal B-mediated renal protection, the protein expression levels of the HPSE/SDC1 axis were detected. The results showed that the protein expression of HPSE, FGF- 2 , TGF- $\beta 1$ and $\alpha$-SMA were significantly increased, while the expression levels of SDC1 and E-cadherin were significantly reduced after modelling compared with those of the control group. However, treatment with Sal B significantly reversed these changes (Fig. 9). Furthermore, the results of immunofluorescence also showed that Sal B could notably reduce the expression level of HPSE and promote the expression level of SDC1 in HK-2 cells after treatment with AngII (Fig. 10). 

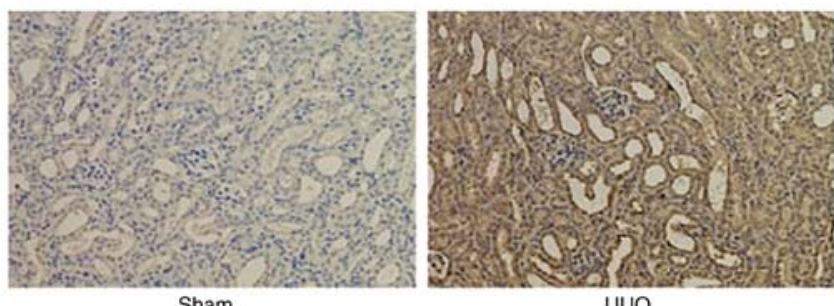

Sham

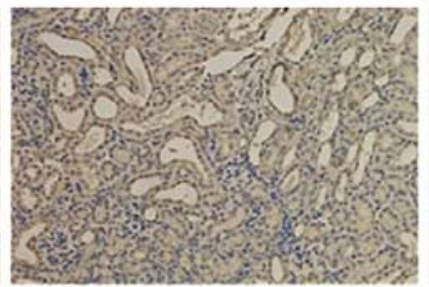

$12.5 \mathrm{mg} / \mathrm{kg}$

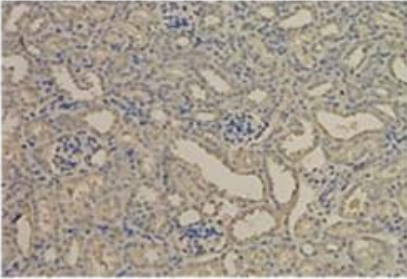

$6.25 \mathrm{mg} / \mathrm{kg}$

HPSE

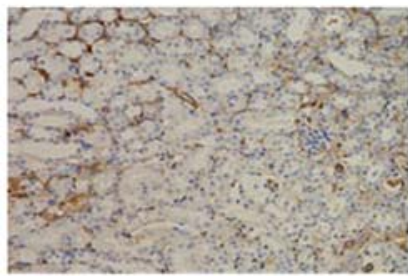

Sham

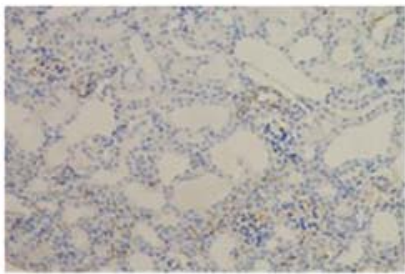

UUO

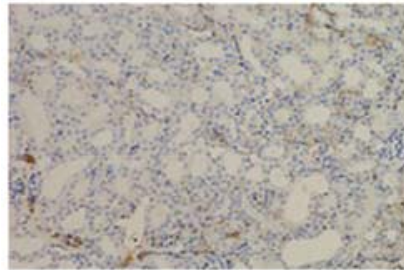

$6.25 \mathrm{mg} / \mathrm{kg}$

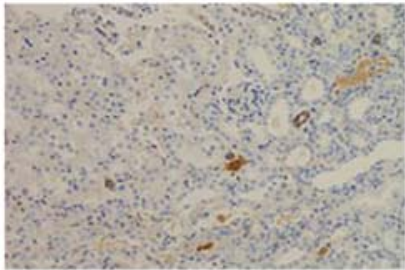

$12.5 \mathrm{mg} / \mathrm{kg}$

SDC1
$25 \mathrm{mg} / \mathrm{kg}$

Figure 5. Immunochemical staining of HPSE and SDC1 $(n=3)$. Salvianolic acid B treatment notably downregulated the expression of HPSE but upregulated the expression of SDC1 in mice kidneys (magnification, x200). UUO, unilateral ureteral obstruction; HPSE, heparinase; SDC1, syndecan-1.
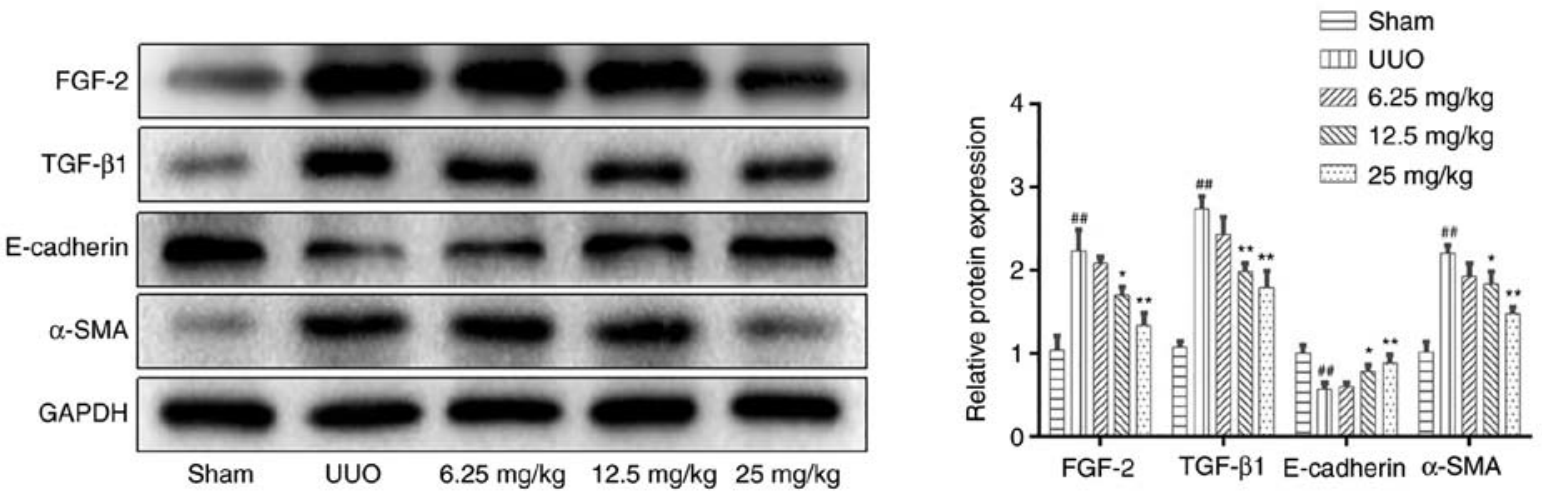

Figure 6. Salvianolic acid B reduces tubular epithelial-myofibroblast transdifferentiation in UUO mice. Western blotting was performed to determine the protein expression levels of FGF-2, TGF- $\beta 1$, E-cadherin and $\alpha$-SMA in the kidney tissues of each group of mice. The expression was normalized to that of GAPDH, and the data were expressed as the mean $\pm \mathrm{SD}$ of 3 independent experiments. ${ }^{*} \mathrm{P}<0.05$ and ${ }^{* *} \mathrm{P}<0.01$ vs. UUO group; ${ }^{\# \#} \mathrm{P}<0.01$ vs. sham group. UUO, unilateral ureteral obstruction; FGF-2, fibroblast growth factor-2; TGF- $\beta 1$, transforming growth factor- $\beta 1 ; \alpha$-SMA, $\alpha$-smooth muscle actin.

Overexpression of the HPSE gene suppresses renal interstitial fibrosis by inhibiting the HPSE/SDC1 axis. In order to further assess the effects of the overexpression of the HPSE gene on renal interstitial fibrosis, the protein expression levels of the HPSE/SDC1 axis were detected using western blotting. The findings showed that overexpression of HPSE could 

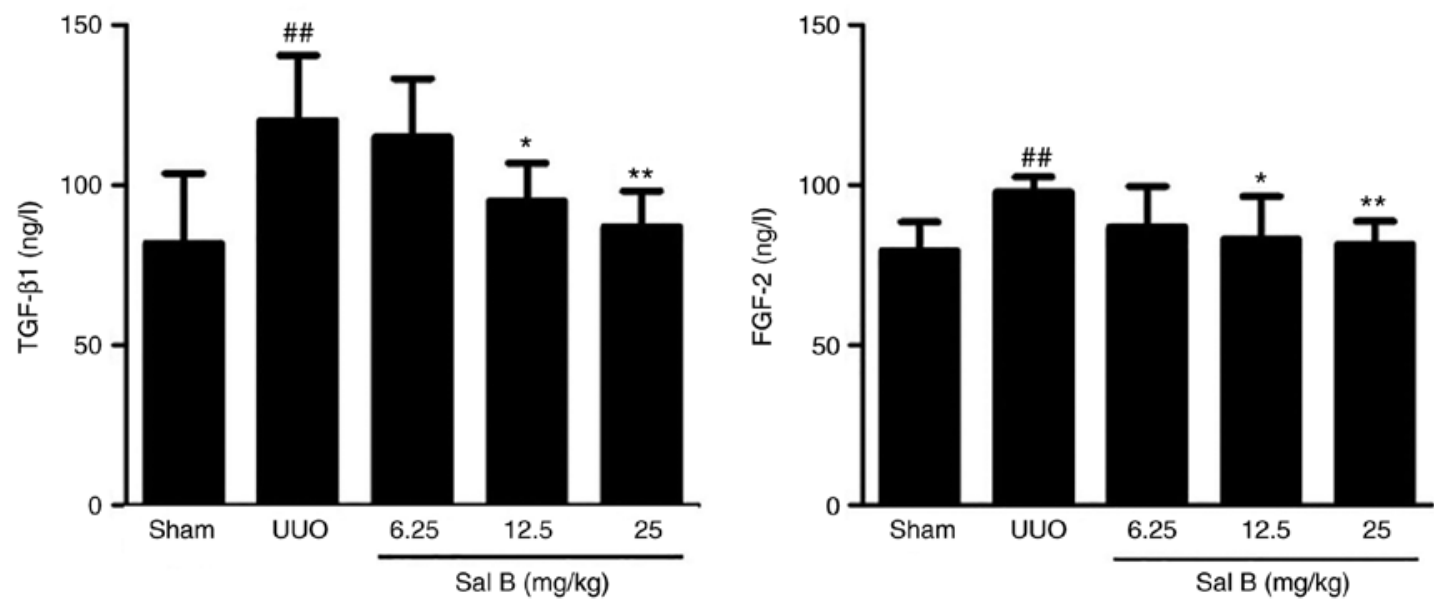

Figure 7. Effect of Sal B on TGF- $\beta 1 /$ FGF-2 in serum ( $\mathrm{n}=3$ mice/group). Mice were intraperitoneally injected with Sal B after left ureteral ligation. Data are expressed as the mean $\pm \mathrm{SD} .{ }^{*} \mathrm{P}<0.05$ and ${ }^{* *} \mathrm{P}<0.01$ vs. UUO group; ${ }^{\# *} \mathrm{P}<0.01$ vs. sham group. Sal $\mathrm{B}$, salvianolic acid B; UUO, unilateral ureteral obstruction; FGF-2, fibroblast growth factor-2; TGF- $\beta 1$, transforming growth factor- $\beta 1$.
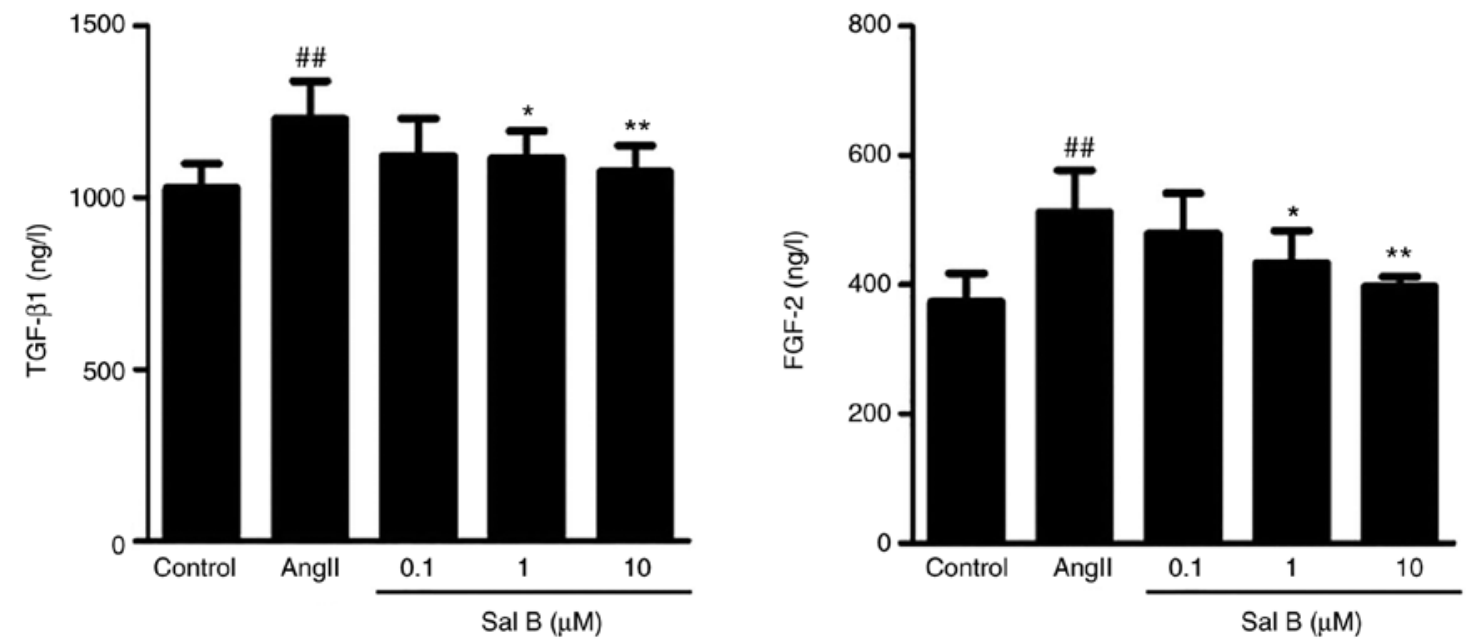

Figure 8. Sal B significantly reduces the expression levels of TGF- $\beta 1$ and FGF-2 in HK-2 cell supernatant $(\mathrm{n}=3)$. Data are expressed as the mean $\pm \mathrm{SD}$. ${ }^{*} \mathrm{P}<0.05$ and ${ }^{* *} \mathrm{P}<0.01$ vs. model group; ${ }^{\# \#} \mathrm{P}<0.01$ vs. control group. Sal B, salvianolic acid B; FGF-2, fibroblast growth factor- 2 ; TGF- $\beta 1$, transforming growth factor- $\beta 1$; AngII, angiotensin II.
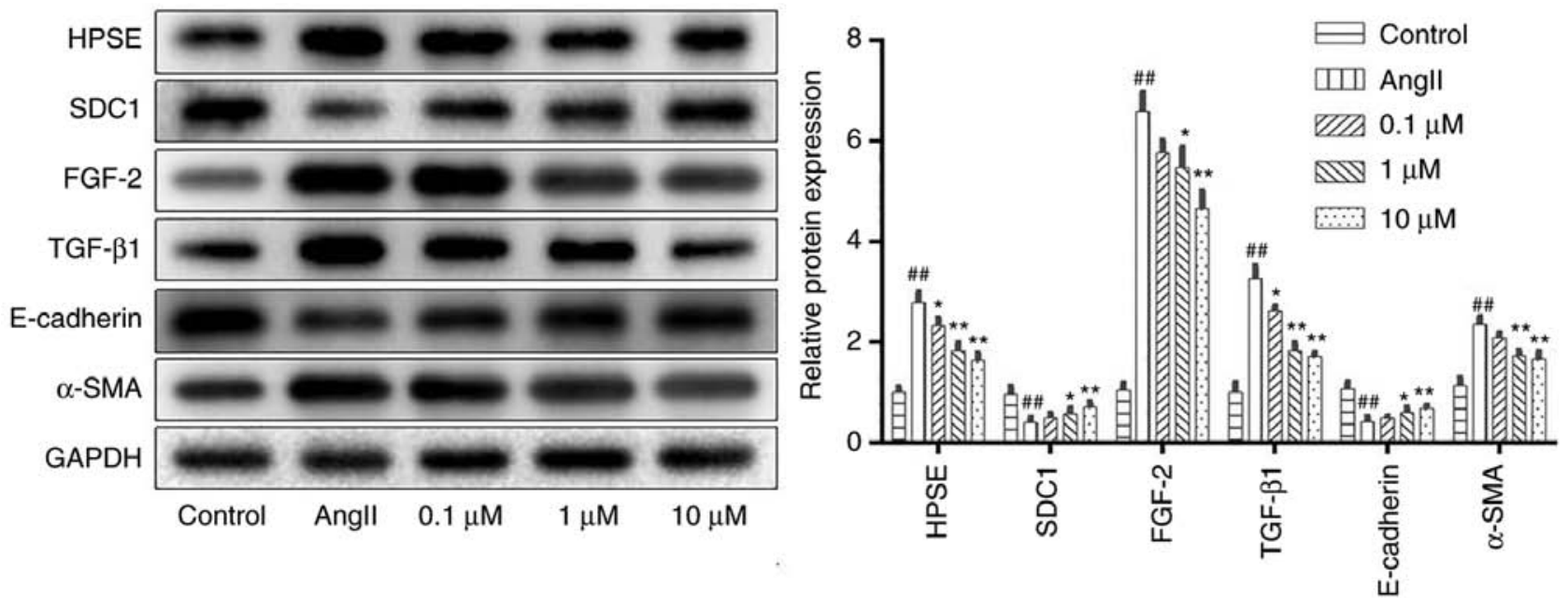

Figure 9. Salvianolic acid B inhibits tubular epithelial-myofibroblast transdifferentiation in HK-2 cells. Western blotting was performed to determine the protein expression levels of HPSE, SDC1, FGF-2, TGF- $\beta 1$, E-cadherin and $\alpha$-SMA in each group. The expression was normalized to that of GAPDH and the data are expressed as the mean $\pm \mathrm{SD}$ of 3 independent experiments. ${ }^{*} \mathrm{P}<0.05$ and ${ }^{* * *} \mathrm{P}<0.01$ vs. model group; ${ }^{\# \#} \mathrm{P}<0.01$ vs. control group. HPSE, heparinase; SDC1, syndecan-1; FGF-2, fibroblast growth factor-2; TGF- $\beta 1$, transforming growth factor- $\beta 1$; $\alpha$-SMA, $\alpha$-smooth muscle actin; AngII, angiotensin II. 

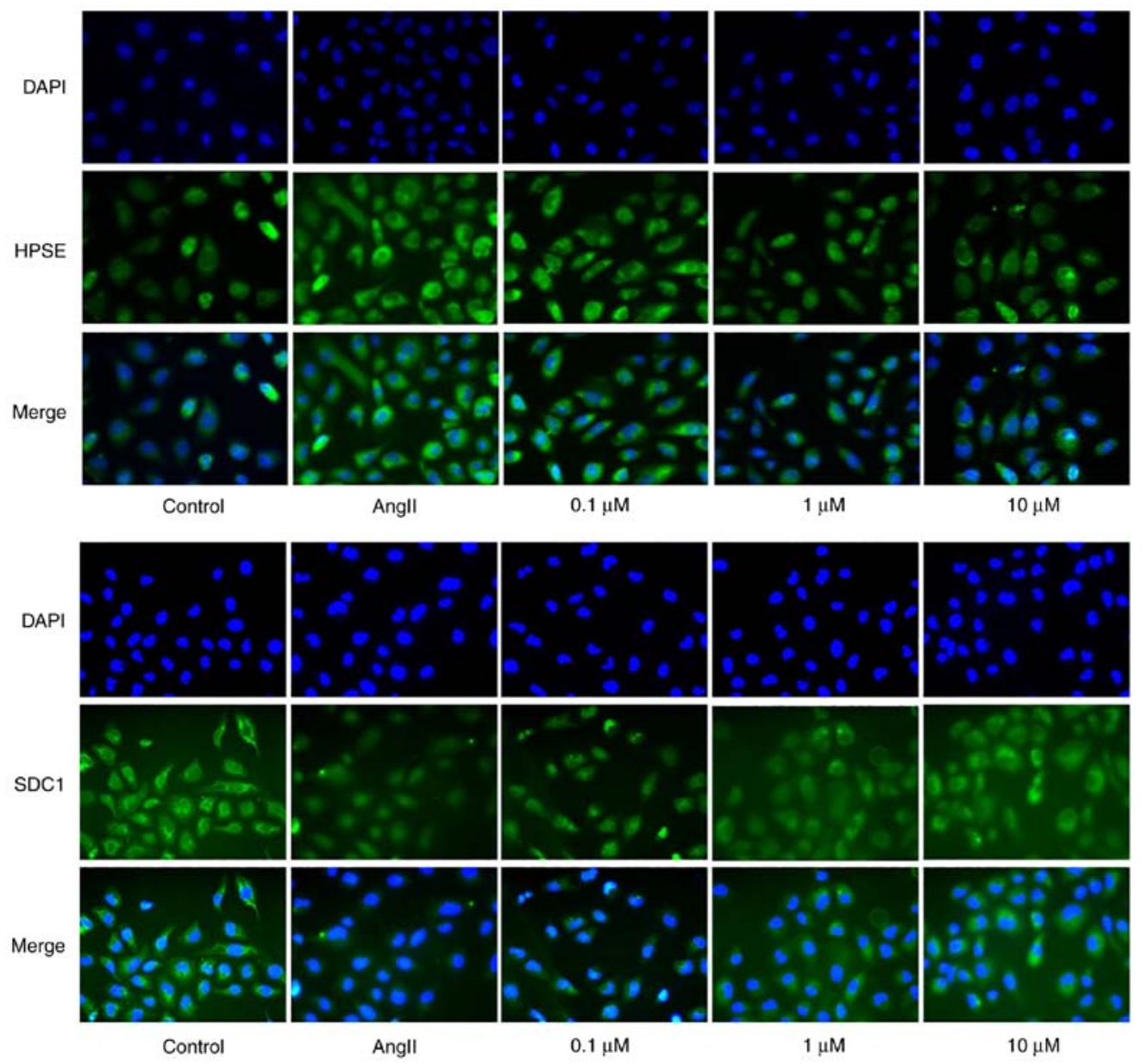

Figure 10. Salvianolic acid B treatment notably downregulates the expression of HPSE and upregulates the expression of SDC1 in HK-2 cells ( $\mathrm{n}=3$ ). HPSE, heparinase; SDC1, syndecan-1; AngII, angiotensin II.
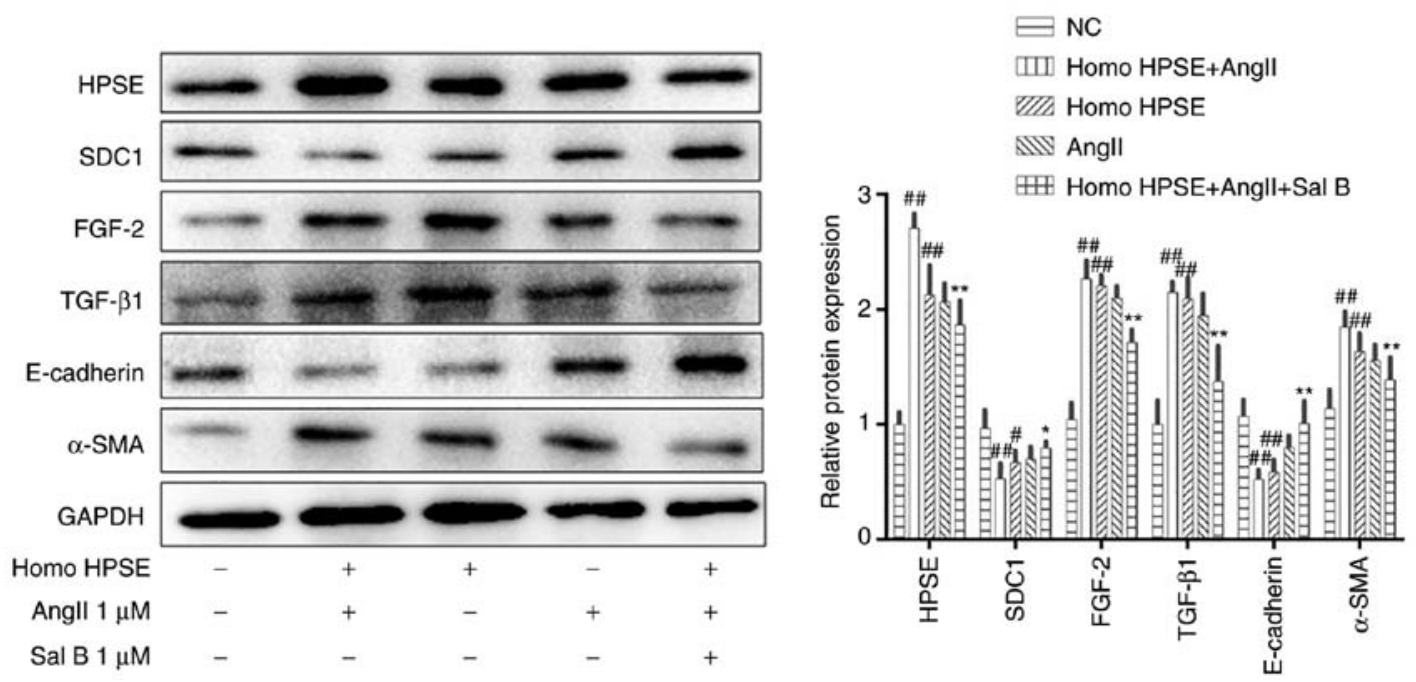

Figure 11. Sal B treatment significantly inhibits the activation of the HPSE/SDC1 axis induced by overexpression of the HPSE gene. The expression was normalized to that of GAPDH, and the data are expressed as the mean \pm SD of 3 independent experiments. ${ }^{*} \mathrm{P}<0.05$ and ${ }^{* *} \mathrm{P}<0.01$ vs. Homo HPSE + AngII group; ${ }^{\#} \mathrm{P}<0.05$ and ${ }^{\# \#} \mathrm{P}<0.01$ vs. NC group. NC, negative control; Sal B, salvianolic acid B; HPSE, heparinase; SDC1, syndecan-1; AngII, angiotensin II; FGF-2, fibroblast growth factor-2; TGF- $\beta 1$, transforming growth factor- $\beta 1 ; \alpha$-SMA, $\alpha$-smooth muscle actin.

significantly upregulate the expression levels of HPSE, FGF-2, TGF- $\beta 1$ and $\alpha$-SMA, and downregulate the expression levels of SDC1 and E-cadherin. However, treatment with Sal B could notably restore these changes (Fig. 11).

\section{Discussion}

Various types of CKDs can lead to renal fibrosis and eventually renal failure; thus, there are numerous studies on the 
factors behind renal fibrosis. AngII is a key mediator that is associated with the development of renal fibrosis $(33,34)$, it has been reported to promote TGF- $\beta 1$ expression, leading to the production of extracellular matrix and inhibition of the extracellular matrix degradation (35). Moreover, TGF- $\beta 1$ has been reported to induce tubular epithelial cells to transdifferentiate into myofibroblasts (36). In the present study, AngII was used to promote the transdifferentiation of HK-2 cells into myoblasts and to promote the expression of TGF- $\beta 1$. The results showed that AngII could promote TEMT via activation of the HPSE/SDC1 axis. However, Sal B treatment notably decreased the expression levels of extracellular matrix and TEMT markers.

TEMT plays an important role in renal interstitial fibrosis. Under the stimulation of numerous factors, renal tubular epithelial cells could release cytokines (such as TGF- $\beta 1$ and FGF-2) into the tubulointerstitial microenvironment, and eventually lead to TEMT $(37,38)$, which is characterized by decreased expression of E-cadherin and increased expression of $\alpha$-SMA (39). Sal B, as one of the main water-soluble components of Salvia miltiorrhiza Bge, has been shown by previous studies to be able to inhibit TEMT by antioxidation (40) and to decrease $\alpha$-SMA expression (41). The present study demonstrated that Sal B treatment could significantly reduce the expression levels of TGF- $\beta 1$ and FGF-2, and regulate the expression of E-cadherin and $\alpha$-SMA.

HS proteoglycans are important constituents of the cell membrane and are associated with multiple physiological processes (42). Previous reports demonstrated that SDC1 in renal tubular epithelial cells is rich in HS chains. HPSE could specifically cleave the HS side chain of SDC1 and lead to the release of several cytokines, such as TGF- $\beta 1$ and FGF-2. The present study showed that Sal B could significantly reduce the expression of HPSE, which indicates that Sal B could attenuate renal interstitial fibrosis by regulating the HPSE/SDC1 axis. However, considering the characteristics of natural products with multiple targets, further in vitro and in vivo research is required to investigate the underlying mechanisms of Sal B in renal interstitial fibrosis.

In summary, the present study demonstrated that activation of the HPSE/SDC1 axis, which promotes TEMT responses and exacerbates renal injury, may play an important role in renal interstitial fibrosis. Sal B, as a natural product with little toxicity, may provide new prospects for the treatment of renal interstitial fibrosis.

\section{Acknowledgements}

Not applicable.

\section{Funding}

This study was supported by Jiangsu Province University Brand Professional Construction Project (grant no. PPZY2015A070).

\section{Availability of data and materials}

The datasets used and/or analyzed during the current study are available from the corresponding author on reasonable request.

\section{Authors' contributions}

YH and MW contributed equally to this work. LX designed the study. YH, MW, YP and QL performed the experiments and analyzed the data. YH, MW and LX contributed to the writing of the manuscript. All authors contributed to the revision of this manuscript and approved the final manuscript.

\section{Ethics approval and consent to participate}

The protocol was approved by the Animal Ethics Committee of Nanjing University of Chinese Medicine (approval no. ACU-2320151203).

\section{Patient consent for publication}

Not applicable.

\section{Competing interests}

The authors declare that they have no competing interests.

\section{References}

1. Levey AS, Eckardt KU, Tsukamoto Y, Levin A, Coresh J, Rossert J, De Zeeuw D, Hostetter TH, Lameire N and Eknoyan G: Definition and classification of chronic kidney disease: A position statement from Kidney Disease: Improving Global Outcomes (KDIGO). Kidney Int 67: 2089-2100, 2005.

2. Parrish AR: Advances in chronic kidney disease. Int J Mol Sci 17: 1314, 2016.

3. Webster AC, Nagler EV, Morton RL and Masson P: Chronic kidney disease. Lancet 389: 1238-1252, 2017.

4. Wilkinson TJ, Shur NF and Smith AC: 'Exercise as medicine' in chronic kidney disease. Scand J Med Sci Sports 26: 985-988, 2016.

5. Li X, Bayliss G and Zhuang S: Cholesterol crystal embolism and chronic kidney disease. Int J Mol Sci 18: 1120, 2017.

6. Humphreys BD: Mechanisms of renal fibrosis. Annu Rev Physiol 80: 309-326, 2018.

7. Li R, Guo Y, Zhang Y, Zhang X, Zhu L and Yan T: Salidroside ameliorates renal interstitial fibrosis by inhibiting the TLR4/NF- $\kappa B$ and MAPK signaling pathways. Int J Mol Sci 20: $1103,2019$.

8. Xie XS, Yang M, Liu HC, Zuo C, Li Z, Deng Y and Fan JM: Influence of ginsenoside $\operatorname{Rg} 1$, a panaxatriol saponin from Panax notoginseng, on renal fibrosis in rats with unilateral ureteral obstruction. J Zhejiang Univ Sci B 9: 885-894, 2008

9. Li JH, Wang W, Huang XR, Oldfield M, Schmidt AM, Cooper ME and Lan HY: Advanced glycation end products induce tubular epithelial-myofibroblast transition through the RAGE-ERK1/2 MAP kinase signaling pathway. Am J Pathol 164: 1389-1397, 2004.

10. Liu JH, He L, Zou ZM, Ding ZC, Zhang X, Wang H, Zhou P, Xie L, Xing S and Yi CZ: A novel inhibitor of homodimerization targeting MyD88 ameliorates renal interstitial fibrosis by counteracting TGF- $\beta 1$-induced EMT in vivo and in vitro. Kidney Blood Press Res 43: 1677-1687, 2018.

11. Waisberg J, Theodoro TR, Matos LL, Orlandi FB, Serrano RL, Saba GT and Pinhal MA: Immunohistochemical expression of heparanase isoforms and syndecan-1 proteins in colorectal adenomas. Eur J Histochem 60: 2590, 2016.

12. Palaiologou M, Delladetsima I and Tiniakos D: CD138 (syndecan-1) expression in health and disease. Histol Histopathol 29: 177-189, 2014.

13. Masola V, Bellin G, Gambaro G and Onisto M: Heparanase: A multitasking protein involved in extracellular matrix (ECM) remodeling and intracellular events. Cells 7: 236, 2018.

14. Jiao W, Chen Y, Song H, Li D, Mei H, Yang F, Fang E, Wang X, Huang K, Zheng L and Tong Q: HPSE enhancer RNA promotes cancer progression through driving chromatin looping and regulating hnRNPU/p300/EGR1/HPSE axis. Oncogene 37: 2728-2745, 2018 
15. Lorente-Gea L, Garcia B, Martin C, Quiros LM and Fernandez-Vega I: Heparan sulfate proteoglycans and heparanases in Alzheimer's disease: Current outlook and potential therapeutic targets. Neural Regen Res 12: 914-915, 2017.

16. Masola V, Zaza G, Onisto M, Lupo A and Gambaro G: Impact of heparanase on renal fibrosis. J Transl Med 13: 181, 2015.

17. Shu T, Pang M, Rong L, Liu C, Wang J, Zhou W, Wang X and Liu B: Protective effects and mechanisms of salvianolic acid B against $\mathrm{H}_{2} \mathrm{O}_{2}$-induced injury in induced pluripotent stem cellderived neural stem cells. Neurochem Res 40: 1133-1143, 2015.

18. Lin C, Liu Z, Lu Y, Yao Y, Zhang Y, Ma Z, Kuai M, Sun X, Sun S, Jing Y, et al: Cardioprotective effect of Salvianolic acid B on acute myocardial infarction by promoting autophagy and neovascularization and inhibiting apoptosis. J Pharm Pharmacol 68: 941-952, 2016.

19. Katary MA, Abdelsayed R, Alhashim A, Abdelhasib M and Elmarakby AA: Salvianolic acid B slows the progression of breast cancer cell growth via enhancement of apoptosis and reduction of oxidative stress, inflammation, and angiogenesis Int J Mol Sci 20: 5653, 2019.

20. Wang B, Sun J, Shi Y and Le G: Salvianolic acid B inhibits high-fat diet-induced inflammation by activating the Nrf2 pathway. J Food Sci 82: 1953-1960, 2017.

21. Wang QL, Tao YY, Yuan JL, Shen L and Liu CH: Salvianolic acid $\mathrm{B}$ prevents epithelial-to-mesenchymal transition through the TGF-beta1 signal transduction pathway in vivo and in vitro. BMC Cell Biol 11: 31, 2010.

22. Hu Y, Li Q, Pan Y and Xu L: Sal B alleviates myocardial ischemic injury by inhibiting TLR4 and the priming phase of NLRP3 inflammasome. Molecules 24: 4416, 2019.

23. Schwalm S, Beyer S, Frey H, Haceni R, Grammatikos G, Thomas D, Geisslinger G, Schaefer L, Huwiler A and Pfeilschifter J: Sphingosine kinase-2 deficiency ameliorates kidney fibrosis by up-regulating smad7 in a mouse model of unilateral ureteral obstruction. Am J Pathol 187: 2413-2429, 2017.

24. Yao Y, Zhang J, Tan DQ, Chen XY, Ye DF, Peng JP, Li JT, Zheng YQ, Fang L, Li YK and Fan MX: Interferon- $\gamma$ improves renal interstitial fibrosis and decreases intrarenal vascular resistance of hydronephrosis in an animal model. Urology 77: 761.e8-e13, 2011

25. Dou F, Liu Y, Liu L, Wang J, Sun T, Mu F, Guo Q, Guo C, Jia N, Liu W, et al: Aloe-emodin ameliorates renal fibrosis via inhibiting PI3K/Akt/mTOR signaling pathway in vivo and in vitro. Rejuvenation Res 22: 218-229, 2019.

26. Masola V, Zaza G, Granata S, Gambaro G, Onisto M and Lupo A: Everolimus-induced epithelial to mesenchymal transition in immortalized human renal proximal tubular epithelial cells: Key role of heparanase. J Transl Med 11: 292, 2013

27. Masola V, Gambaro G, Tibaldi E, Brunati AM, Gastaldello A D'Angelo A, Onisto M and Lupo A: Heparanase and syndecan-1 interplay orchestrates fibroblast growth factor-2-induced epithelial-mesenchymal transition in renal tubular cells. J Biol Chem 287: 1478-1488, 2012.

28. Takahashi M, Takayama S, Suga H, Kadomura S, Kojima M, Iwao K, Takeda K, Sato H, Kobayashi M and Saitoh H: Factors resulting correlation and differences in renal function evaluation index using the serum cystatin $\mathrm{c}$ and creatinine as measured by an enzymatic method. Yakugaku Zasshi 140: 81-90, 2020 (In Japanese).
29. Sun YB, Qu X, Caruana G and Li J: The origin of renal fibroblasts/myofibroblasts and the signals that trigger fibrosis. Differentiation 92: 102-107, 2016.

30. Wang HY, Zhang C, Xiao QF, Dou HC, Chen Y, Gu CM and Cui MJ: Hepatocyte growth factor inhibits tubular epithelial-myofibroblast transdifferentiation by suppression of angiotensin II via the JAK2/STAT3 signaling pathway. Mol Med Rep 15: 2737-2743, 2017.

31. Li X, Li X, Zhang Q and Zhao T: Low molecular weight fucoidan and its fractions inhibit renal epithelial mesenchymal transition induced by TGF- $\beta 1$ or FGF-2. Int J Biol Macromol 105: 1482-1490, 2017.

32. Yuan Q, Tan RJ and Liu Y: Myofibroblast in kidney fibrosis: Origin, activation, and regulation. Adv Exp Med Biol 1165: 253-283, 2019.

33. Liu M, Ning X, Li R, Yang Z, Yang X, Sun S and Qian Q: Signalling pathways involved in hypoxia-induced renal fibrosis. J Cell Mol Med 21: 1248-1259, 2017.

34. Yu X, Xia Y, Zeng L, Zhang X, Chen L, Yan S, Zhang R, Zhao C, Zeng Z, Shu Y, et al: A blockade of PI3K $\gamma$ signaling effectively mitigates angiotensin II-induced renal injury and fibrosis in a mouse model. Sci Rep 8: 10988, 2018.

35. Fujimura K, Wakino S, Minakuchi H, Hasegawa K, Hosoya K, Komatsu M, Kaneko Y, Shinozuka K, Washida N, Kanda T, et al: Ghrelin protects against renal damages induced by angiotensin-II via an antioxidative stress mechanism in mice. PLoS One 9: e94373, 2014

36. Thakur S, Viswanadhapalli S, Kopp JB, Shi Q, Barnes JL, Block K, Gorin Y and Abboud HE: Activation of AMP-activated protein kinase prevents TGF- $\beta 1$-induced epithelial-mesenchymal transition and myofibroblast activation. Am J Pathol 185: 2168-2180, 2015.

37. Rodriguez-Mateo C, Torres B, Gutierrez G and Pintor-Toro JA: Downregulation of Lnc-Spry1 mediates TGF- $\beta$-induced epithelial-mesenchymal transition by transcriptional and posttranscriptional regulatory mechanisms. Cell Death Differ 24: 785-797, 2017.

38. Griggs LA, Hassan NT, Malik RS, Griffin BP, Martinez BA, Elmore LW and Lemmon CA: Fibronectin fibrils regulate TGF- $\beta 1$-induced Epithelial-mesenchymal transition. Matrix Biol 60-61: 157-175, 2017.

39. Lu HY, Zhou J, Lu M, Liu YM, Wang F, Lin M and Zhang Y: Protection and mechanisms of salvianolic-acid B on experimental renal interstitial fibrosis in rats. Zhong Yao Cai 33: 1755-1759, 2010 (In Chinese).

40. Wang QL, Yuan JL, Tao YY, Hu YY and Liu CH: Effect of salvianolic acid $\mathrm{B}$ on renal interstitial fibrosis in rats induced by $\mathrm{HgCl}$ 2. Pharmacol Clin Chin Materia Medica 24: 12-15, 2008

41. Zhou J, Zhang Y, Lu H, Liu Y and Lin M: Effect of salvianolic acid $B$ on generation and activation of myofibroblast in rat with renal interstitial fibrosis. Zhongguo Zhong Yao Za Zhi 34: 2790-2793, 2009 (In Chinese).

42. Motta G and Tersariol ILS: Modulation of the plasma kallikrein-kinin system proteins performed by heparan sulfate proteoglycans. Front Physiol 8: 481, 2017.

This work is licensed under a Creative Commons Attribution-NonCommercial-NoDerivatives 4.0 International (CC BY-NC-ND 4.0) License. 